\title{
Charge and Spin Density Perturbation on Iron Nuclei by Non-Magnetic Impurities Substituted on the Iron Sites in $\alpha$-Fe
}

\author{
A. B£ACHOWSKI* \\ Mössbauer Spectroscopy Division, Institute of Physics \\ Pedagogical University \\ Podchorążych 2, PL-30-084 Kraków, Poland
}

\begin{abstract}
The paper is aimed at the review of the charge and spin density perturbation on the iron nucleus in the bcc iron-based binary alloys containing as the impurity either $4 d(\mathrm{Nb}, \mathrm{Mo}, \mathrm{Ru}, \mathrm{Rh}, \mathrm{Pd})$ or $5 d(\mathrm{Os}, \mathrm{Ir}, \mathrm{Au})$ metals. Additionally, Ga was used as such impurity as well. Measurements were performed by means of the ${ }^{57} \mathrm{Fe}$ transmission Mössbauer spectroscopy at room temperature. Powder X-ray diffraction data for alloys investigated show linear dependence of the lattice constant versus impurity concentration. The Mössbauer data were treated assuming random distribution of the impurity over the iron sites and additive effect for the charge density perturbation, and additive in the algebraic sense effect for the corresponding spin density perturbation. Hence, the effect of impurity depends solely on the distance between impurity and the iron nucleus under above assumptions. It has been found that impurities being further away than a third or in some cases as the second neighbor do not contribute directly to the charge and spin perturbation. On the other hand, they have usually some minor effect on the average charge and spin density. Generally, the perturbation to either charge or spin density has some oscillatory character versus distance from the impurity. The phase and period of the charge oscillation is vastly different from the phase and period of the spin oscillation in the majority of cases. Substitution of the impurities with the increasing number of $4 d$ or $5 d$ electrons leads to the lowering of the electron density on the iron nucleus and causes decreased band spin density on this nucleus. Subsequent impurities donate more and more $d$-type electrons to the band, and the latter screen more and more effectively $s$-like electrons. Hence, the density of the $s$-like electrons on the iron nucleus diminishes. Impurities with $5 d$ electrons have generally stronger effect on the charge and spin density perturbation than impurities with $4 d$ electrons.
\end{abstract}

PACS numbers: 75.50.Bb, 76.80.+y

*e-mail: sfblacho@cyf-kr.edu.pl 


\section{Introduction}

Iron crystallizes in the bcc structure below $912^{\circ} \mathrm{C}$ and under low pressure. This phase orders in the ferromagnetic fashion at relatively high temperature of $770^{\circ} \mathrm{C}$ and the magnetically ordered iron having bcc structure is called $\alpha$-Fe [1]. Pure iron is extremely soft magnet with the easy axis of magnetization oriented along one of the principal axes of the chemical unit cell. Hence, the internal hyperfine field on the iron nucleus is almost entirely due to the Fermi contact term, as dipolar fields cancel exactly for the pure iron, while the orbital contribution is almost quenched in the metallic environment of iron and for the $3 d$ magnetic shell. On the other hand, magnetic moments are well localized in iron [2]. Hence, the hyperfine field is due to the core polarization and fields transferred from the neighbors via the conduction band. A transferred field is due to the $s$-like electrons as the iron is not heavy enough to have significant contribution from the relativistic $p$-type electrons. The electric field gradient vanishes on the iron nuclei due to the cubic symmetry. Electrons of the $s$-like type generate charge (electron) density on iron nuclei. They belong either to the atomic core or to the conduction band. A contribution due to the relativistic $p$-type electrons is again negligible. Electrons of the higher angular moments practically contribute neither to the charge nor to the spin density on the iron nucleus. One can calculate by using ab initio method absolute second order Doppler (SOD) shift for ${ }^{57} \mathrm{Fe}$ in $\alpha$-Fe. It appears to be $-0.11 \mathrm{~mm} / \mathrm{s}$ in the ground state, and hence the equivalent Debye temperature for SOD amounts to $\theta_{\mathrm{D}}=399 \mathrm{~K}[3]$. Electronic and magnetic properties at room temperature are close to the corresponding properties in the ground state.

Many metallic non-magnetic impurities could be dissolved in the $\alpha$-Fe on the regular iron sites leading to the binary alloys of the random order $[4,5]$. Such impurities do not affect significantly lattice dynamics provided they are sufficiently diluted. They do not generate significant electric field gradients as well due to the effective screening by the conduction band. They could lead to some residual dipolar hyperfine fields on iron nuclei, by these fields are negligible in comparison with the Fermi contact fields. Due to the fact that the system remains highly metallic and cubic no orbital terms are created on iron atoms. Magnetism here is due to the $3 d$ electrons of iron, the latter being sufficiently close to the Fermi surface of the conduction band in order to quench orbital terms. The ferromagnetism of $\alpha$-Fe is preserved as well for sufficiently diluted impurities. On the other hand, the charge (electron) density on iron nuclei is perturbed, and the transferred contact field on iron nuclei is perturbed as well due to the perturbation of the spin density in the conduction band. A perturbation due to the isolated impurity fades away while moving away from the impurity.

Present contribution is aimed at the systematic review of the charge and spin density perturbation on iron nuclei caused by various impurities in the binary systems $\mathrm{Fe}_{1-c} \mathrm{X}_{c}(\mathrm{X}=\mathrm{Nb}$ [6], Mo, Ru [7], Rh, Pd [8], Os [9], Ir [10], Au [11], $\mathrm{Ga}[12])$ of the above type. Here the symbol $c$ stands for the average impurity 
concentration. Samples have been prepared by arc melting of the pure elements. Random distribution of impurities over the iron sites is promoted by the rapid cooling of the ingots having mass of about $1.5 \mathrm{~g}$. Initial cooling rate could be estimated as being more than $500 \mathrm{~K} / \mathrm{s}$. Powder X-ray diffraction data obtained for the quenched alloys show linear behavior of the lattice constant versus impurity concentration.

Results have been obtained by means of the $14.4 \mathrm{keV}$ transmission Mössbauer spectroscopy in ${ }^{57} \mathrm{Fe}$ applying random absorbers kept at room temperature. Single line unpolarized and thin for resonant self-absorption source of ${ }^{57} \mathrm{Co}(\mathrm{Rh})$ was used. All shifts are reported versus shift in the room temperature $\alpha$-Fe. The source was kept at room temperature as well. The MsAa-3 spectrometer was used with 4096 data channels per unfolded spectrum [13].

\section{Consistent model for the data treatment}

Even for diluted impurities one has very many possible configurations around the resonant iron nucleus. Hence, some assumptions leading to the subsequent approximations are necessary. First of all it is assumed that impurities are randomly distributed around the particular resonant nucleus. Furthermore, it is assumed that the perturbation due to the particular impurity scales solely with the effective distance between the resonant nucleus and the impurity. Hence, one can use subsequent coordination shells to describe perturbations. Particular perturbations are treated as additive in the algebraic sense for charge and spin density perturbations both, and the individual perturbation is distinguished to the third coordination shell at most. The number of parameters is reduced significantly under above assumptions despite the fact that the Mössbauer absorption profile is composed of the very large number of sub-profiles. The parameters completely describing scalar hyperfine interactions are as follows: $S_{0}^{(\sigma)}$ describing total (relative isomer) shift due to the all atoms located beyond the last coordination shell $\sigma$ taken into account, and $\Delta S_{s}^{(\sigma)}$ standing for a contribution to the shift due to the single impurity located in the coordination shell $s=1, \ldots, \sigma$. Corresponding parameters describing dipolar magnetic hyperfine interactions, i.e., spin density perturbation are $B_{0}^{(\sigma)}$ and $\Delta B_{s}^{(\sigma)}$. Hence, a complex distribution is described completely by $2(\sigma+1)$ parameters. The impurity concentration $c$ could be used as the additional free parameter in description, but usually it is determined independently. The above model could be easily inserted into the transmission integral algorithm with further allowances particularly for the partial absorber global magnetization. A complete description of the model could be found in Refs. [6, 8]. One can calculate all dependent parameters relying on the parameters described above provided a group symmetry of the lattice is known ( $\operatorname{Im} \overline{3} m$ for systems investigated here). In particular one can calculate respective averages $\langle S\rangle_{\sigma}$ and $\langle B\rangle_{\sigma}$. Recently the isomer shift calibration constant for the $14.4 \mathrm{keV}$ transition in ${ }^{57} \mathrm{Fe}$ has been determined precisely as $a=-0.291 \mathrm{~mm} \mathrm{~s}^{-1}$ a.u. ${ }^{3}$ [3]. Therefore perturbations of 
the isomer shift could be readily transformed into perturbations of the electron density on the resonant nucleus.

\section{Discussion of results}

It has been confirmed by means of the X-ray diffraction that samples are single $\operatorname{Im} \overline{3} m$ phase within impurity ranges discussed here. For $\mathrm{Fe}_{1-c} \mathrm{Mo}_{c}$ and $\mathrm{Fe}_{1-c} \mathrm{Rh}_{c}$ samples traces of iron oxides have been found and taken into account during analysis of the Mössbauer spectra. Lattice constant $a$ and half-width of the $\langle 110\rangle$ Bragg peak have been investigated systematically for $\mathrm{Fe}_{1-c} \mathrm{Os}_{c}$ and $\mathrm{Fe}_{1-c} \mathrm{Au}_{c}$ [11] systems versus impurity concentration $c$. A linear behavior of the lattice constant versus $c$ indicates fair randomness of the alloy within the concentration range investigated. Results for the $\mathrm{Fe}_{1-c} \mathrm{Os}_{c}$ system are shown in Fig. 1. Powder X-ray
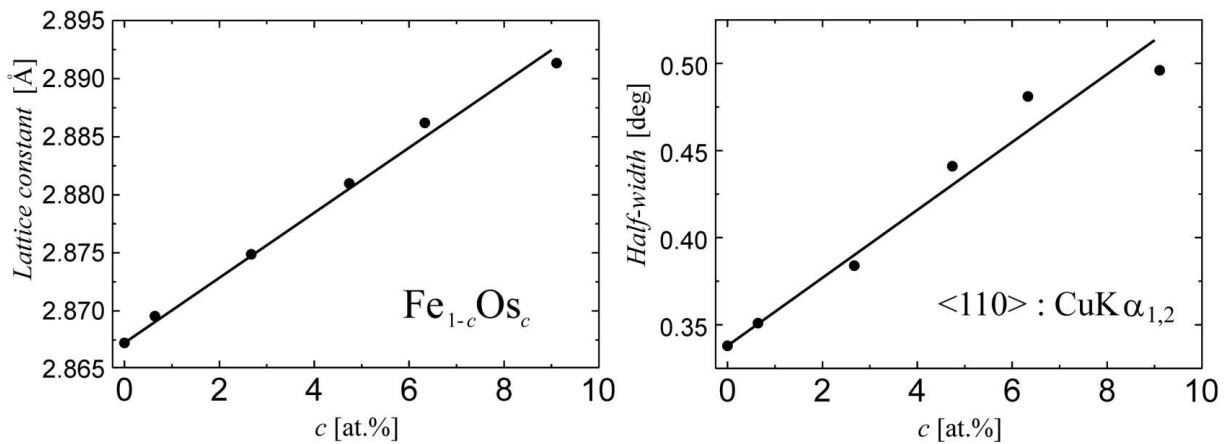

Fig. 1. Lattice constant and half-width of the $\langle 110\rangle$ Bragg peak plotted versus osmium concentration $c$.

data show linear dependence of the lattice constant versus impurity concentration with $\mathrm{d} a / \mathrm{d} c=2.8 \times 10^{-3} \AA /$ at.\%. The Bragg peaks broaden with addition of the impurity due to the lattice relaxation, but they do not split. Usually the impurity concentration limit is higher here than for the equilibrium alloys due to the rapid quench of the molten state. We were able e.g. to dissolve randomly about 9 at.\% of osmium [9], while the equilibrium limit is about 3 at.\% [1]. Hence, alloys investigated here remain in the metastable state retained from the high temperature equilibrium.

The Mössbauer spectra of the $\mathrm{Fe}_{1-c} \mathrm{X}_{c}$ alloys obtained close to the respective impurity solubility limit are shown in Fig. 2. Essential results are summarized in Table. Parameters $\Delta S_{s}^{(\sigma)}$ and $\Delta B_{s}^{(\sigma)}$ are averaged over respective impurity concentrations, i.e., they read as $\left\langle\Delta S_{s}^{(\sigma)}\right\rangle_{c}$ and $\left\langle\Delta B_{s}^{(\sigma)}\right\rangle_{c}$, respectively. Figure 3 shows correlation between $\mathrm{d}\langle B\rangle_{\sigma} / \mathrm{d} c$ and $\mathrm{d}\langle S\rangle_{\sigma} / \mathrm{d} c$ for various systems. One can see approximate linear relationship between these quantities within particular groups of impurities, i.e., for the $4 d$ and $5 d$ impurities. Substitution of the impurities with the increasing number of $4 d$ or $5 d$ electrons leads to the lowering of the electron density on the iron nucleus and causes decreased band spin density on this nu- 

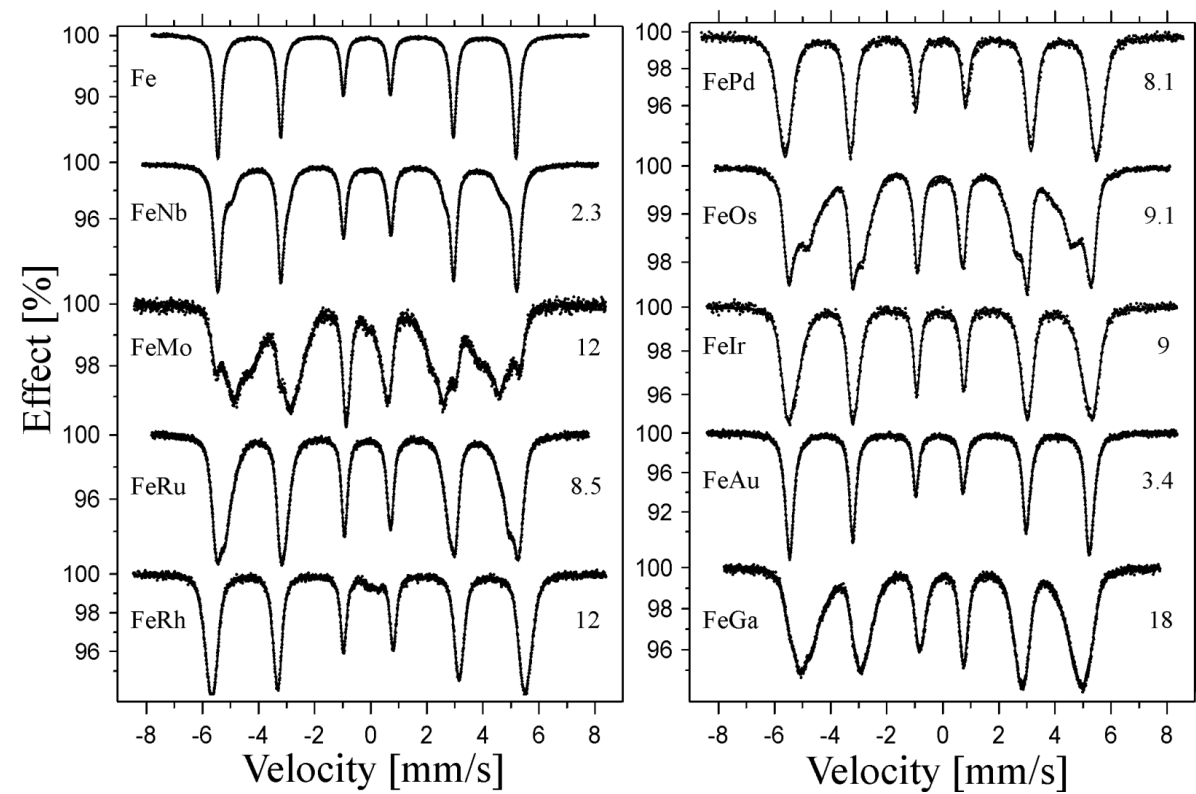

Fig. 2. Mössbauer spectra of the $\mathrm{Fe}_{1-c} \mathrm{X}_{c}$ alloys close to the solubility limit of the component $\mathrm{X}$ with the concentration $c$ shown in the right columns in at.\%.

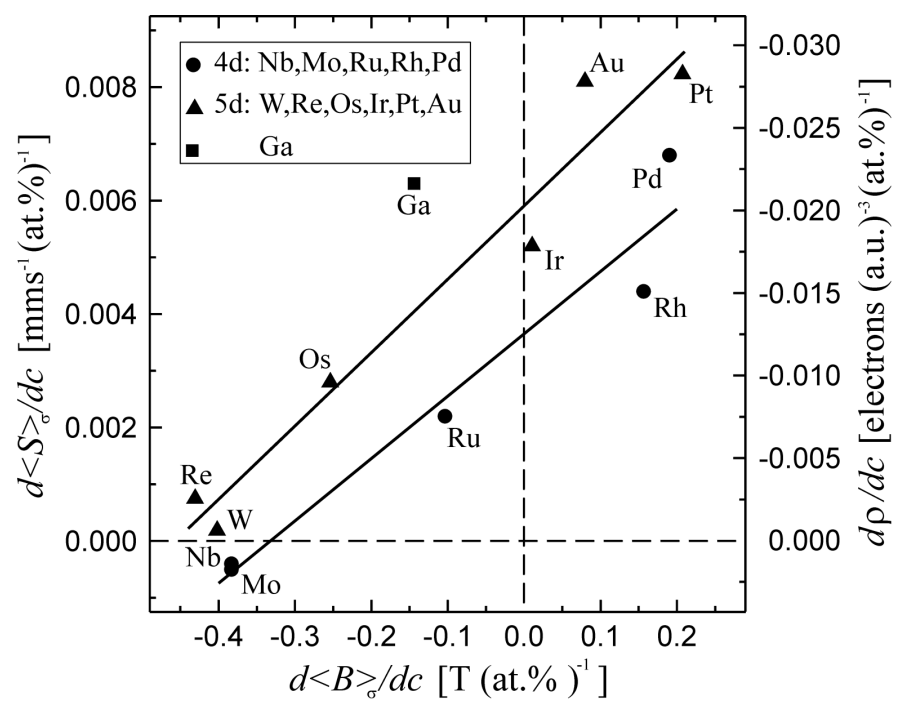

Fig. 3. Correlation between average hyperfine magnetic field change per at. $\%$ of impurity and corresponding change of the isomer shift. Right hand axis is described in the respective change $\mathrm{d} \rho / \mathrm{d} c$ of the electron density $\rho$ on the iron nucleus by using recently determined isomer shift calibration constant. Data for $\mathrm{W}, \mathrm{Re}$, and $\mathrm{Pt}$ are taken from Refs. [14], [15], and [16], respectively. 
TABLE

Essential results for $\mathrm{Fe}_{1-c} \mathrm{X}_{c}$ alloys investigated. Empty fields indicate that $\sigma=2$ model has been applied. Parameters $\Delta S_{s}^{(\sigma)}$ and $\Delta B_{s}^{(\sigma)}$ are averaged over respective impurity concentrations.

\begin{tabular}{|c|c|c|c|c|c|}
\hline \multirow{2}{*}{$\begin{array}{c}\text { Impurity } \\
\mathrm{X}\end{array}$} & $\frac{\mathrm{d}\langle B\rangle_{\sigma}}{\mathrm{d} c}$ & $\frac{\mathrm{d} B_{0}^{(\sigma)}}{\mathrm{d} c}$ & $\Delta B_{1}^{(\sigma)}$ & $\Delta B_{2}^{(\sigma)}$ & $\Delta B_{3}^{(\sigma)}$ \\
\hline & \multicolumn{2}{|c|}{$\left[\mathrm{T}(\text { at. } \%)^{-1}\right]$} & \multicolumn{3}{|c|}{$[\mathrm{T}]$} \\
\hline $\mathrm{Nb}[6]$ & -0.3830 & +0.0381 & -3.55 & -2.30 & \\
\hline Mo & -0.3834 & +0.0170 & -4.18 & -2.30 & +0.51 \\
\hline $\mathrm{Ru}[7]$ & -0.1039 & +0.0014 & -1.99 & -0.09 & +0.52 \\
\hline $\mathrm{Rh}$ & +0.1561 & +0.0040 & +0.73 & +0.70 & +0.43 \\
\hline $\mathrm{Pd}[8]$ & +0.1902 & +0.0052 & +1.38 & +1.05 & +0.67 \\
\hline Os [9] & -0.2537 & +0.0927 & -3.97 & -1.11 & \\
\hline $\operatorname{Ir}[10]$ & +0.0104 & -0.0107 & +0.66 & -1.88 & +0.70 \\
\hline $\mathrm{Au}[11]$ & +0.0799 & +0.0121 & +0.86 & 0 & \\
\hline $\mathrm{Ga}[12]$ & -0.1439 & -0.0271 & -2.02 & -0.02 & +0.44 \\
\hline \multirow{2}{*}{$\begin{array}{c}\text { Impurity } \\
\mathrm{X}\end{array}$} & $\frac{\mathrm{d}\langle S\rangle_{\sigma}}{\mathrm{d} c}$ & $\frac{\mathrm{d} S_{0}^{(\sigma)}}{\mathrm{d} c}$ & $\Delta S_{1}^{(\sigma)}$ & $\Delta S_{2}^{(\sigma)}$ & $\Delta S_{3}^{(\sigma)}$ \\
\hline & \multicolumn{2}{|c|}{$[\mathrm{mm} /(\mathrm{s}$ at. $\%)]$} & \multicolumn{3}{|c|}{$[\mathrm{mm} / \mathrm{s}]$} \\
\hline $\mathrm{Nb}[6]$ & -0.0004 & +0.0025 & -0.033 & -0.015 & \\
\hline Mo & -0.0005 & +0.0021 & -0.033 & -0.005 & +0.003 \\
\hline $\mathrm{Ru}[7]$ & +0.0022 & -0.0010 & -0.019 & +0.072 & +0.005 \\
\hline $\mathrm{Rh}$ & +0.0044 & +0.0011 & -0.015 & -0.005 & +0.039 \\
\hline $\mathrm{Pd}[8]$ & +0.0068 & +0.0060 & +0.010 & +0.008 & +0.003 \\
\hline Os [9] & +0.0028 & +0.0032 & -0.031 & +0.014 & \\
\hline Ir $[10]$ & +0.0052 & +0.0031 & -0.031 & +0.003 & +0.033 \\
\hline $\mathrm{Au}[11]$ & +0.0081 & +0.0004 & +0.013 & +0.111 & \\
\hline $\mathrm{Ga}[12]$ & +0.0063 & -0.0004 & +0.036 & +0.076 & -0.002 \\
\hline
\end{tabular}

cleus. Hence, the transferred field is reduced, while the total field is increased, as the almost constant core field is oriented in the opposite way to the transferred field. Subsequent impurities donate more and more $d$-type electrons to the band, and the latter screen more and more effectively $s$-like electrons, the latter giving contribution to the electron density on the nucleus. Hence, the density of the $s$-like electrons on the iron nucleus diminishes. Impurities with $5 d$ electrons have generally stronger effect on the charge and spin density perturbation than impurities with $4 d$ electrons, as $5 d$ electrons are much closer to the Fermi level than $4 d$ electrons.

Parameters $\mathrm{d} B_{0}^{(\sigma)} / \mathrm{d} c$ and $\mathrm{d} S_{0}^{(\sigma)} / \mathrm{d} c$ are small in the absolute terms. This is an indication that isolated impurity is unable to make a significant perturbation of either charge or spin density beyond the $\sigma=3$ coordination shell. 

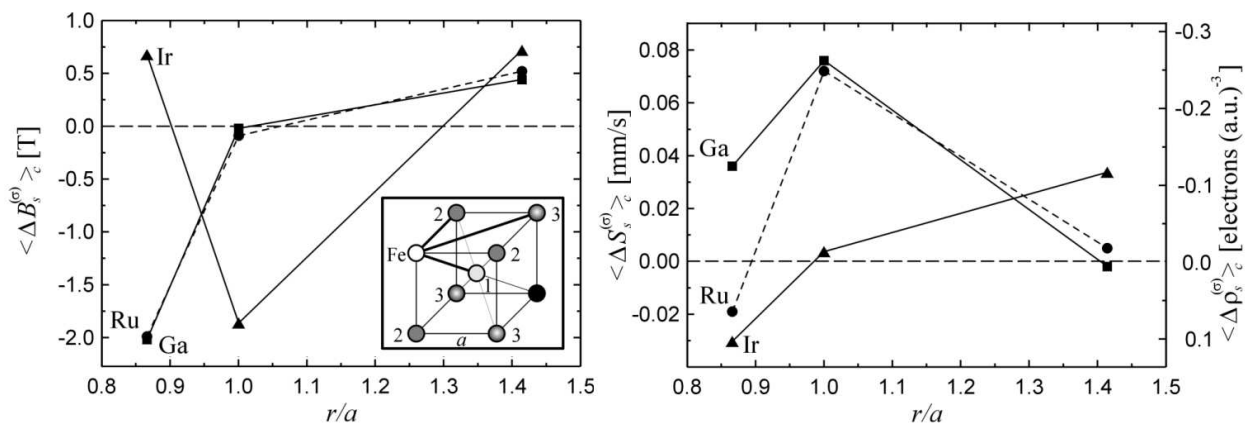

Fig. 4. Average perturbations $\left\langle\Delta S_{s}^{(\sigma)}\right\rangle_{c}$ and $\left\langle\Delta B_{s}^{(\sigma)}\right\rangle_{c}$ plotted for Ru, Ir and Ga impurities versus relative distance from the iron nucleus $r / a$, where $a$ denotes the lattice constant. Lines connecting points are eye guides solely. Corresponding perturbation of the electron density on the iron nucleus $\left\langle\Delta \rho_{s}^{(\sigma)}\right\rangle_{c}$ is shown on the right axis. Inset shows respective coordination shells in the bcc structure around iron atom with the index $s$ shown.

Figure 4 shows parameters $\left\langle\Delta S_{s}^{(\sigma)}\right\rangle_{c}$ and $\left\langle\Delta B_{s}^{(\sigma)}\right\rangle_{c}$ plotted in the case of $\mathrm{Ru}$, Ir and $\mathrm{Ga}$ impurities versus relative (effective) distance $r / a$ from the resonant iron nucleus. One can see strong oscillations of these quantities with respect to the distance between impurity and the probing nucleus. Phases and periods of the respective charge and spin fluctuations seem mutually incommensurate.

In order to investigate global effects of the charge and spin perturbations one can define the following quantities:

$$
\delta S=\sqrt{\sum_{s=1}^{\sigma}\left[\left\langle\Delta S_{s}^{(\sigma)}\right\rangle_{c}\right]^{2}}, \quad \delta B=\sqrt{\sum_{s=1}^{\sigma}\left[\left\langle\Delta B_{s}^{(\sigma)}\right\rangle_{c}\right]^{2}} .
$$

Correlation between dispersions $\delta S$ and $\delta B$ is plotted in Fig. 5. The point $(0,0)$ represents pure $\alpha$-Fe. All alloys investigated fall within the triangle shown in Fig. 5 . Gold lies at the apex with the strongest influence on the charge density perturbation and the smallest influence on the spin density perturbation. On the other hand, molybdenum has the strongest influence on the spin density perturbation. Due to the oscillatory character of the perturbations there is no simple correlation between local charge and/or spin density perturbation and the electronic structure of the impurity.

Heat formation of the binary alloys has been investigated previously by many methods including such microscopic methods like perturbed angular correlations $[17,18]$ and the Mössbauer spectroscopy [19, 20]. Generally binding energies of the impurity-impurity pair are small enough to assure randomness of the alloy quenched from the molten state. Such pairs (or anti-pairs) were observed upon performing prolonged annealing at intermediate temperatures. 


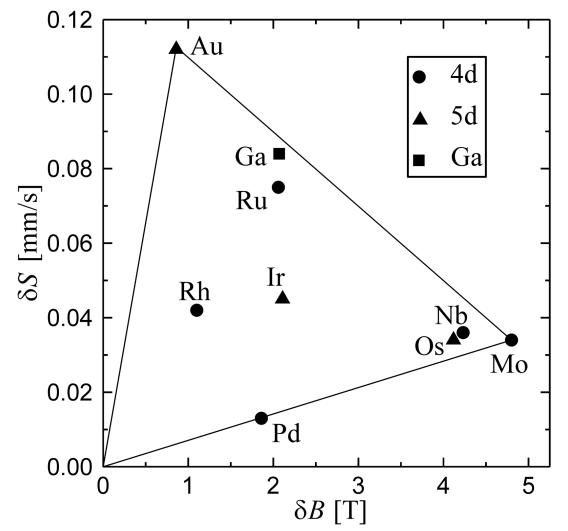

Fig. 5. Correlation between dispersions $\delta S$ and $\delta B$ for systems investigated.

The systems considered here were investigated previously by various authors. A review is given in Ref. [5]. However concentration ranges of impurities were usually very limited (one or two concentrations), and typically only average hyperfine parameters were determined. In a few cases first and occasionally second neighbor impurity effect has been taken into account. In order to determine effect due to the impurity in various coordination shells one needs some model relying on few adjustable parameters, as otherwise one cannot reliably process experimental data.

\section{Acknowledgments}

Professor Krzysztof Ruebenbauer, Pedagogical University, Kraków, Poland is thanked for contribution to the data evaluation and models development. Dr. Jan Żukrowski, AGH University of Science and Technology, Kraków, Poland is thanked for alloys preparation. Dr. Janusz Przewoźnik, AGH University of Science and Technology, Kraków, Poland is acknowledged for performing X-ray diffraction measurements.

\section{References}

[1] T.B. Massalski, Binary Alloys Phase Diagrams, 2nd ed., ASM International, Materials Park, Ohio, USA 1990.

[2] A.H. Morrish, Physical Principles of Magnetism, Wiley-IEEE, New York 2001.

[3] U.D. Wdowik, K. Ruebenbauer, Phys. Rev. B 76, 155118 (2007).

[4] J. Cieślak, S.M. Dubiel, J. Alloys Comp. 350, 17 (2003).

[5] I. Vincze, I.A. Campbell, J. Phys. F, Metal Phys. 3, 647 (1973).

[6] A. Błachowski, K. Ruebenbauer, J. Żukrowski, Phys. Status Solidi B 242, 3201 (2005).

[7] A. Błachowski, K. Ruebenbauer, J. Żukrowski, Phys. Rev. B 73, 104423 (2006). 
[8] A. Błachowski, K. Ruebenbauer, J. Żukrowski, Phys. Scr. 70, 368 (2004).

[9] A. Błachowski, K. Ruebenbauer, J. Żukrowski, Nukleonika 49, S67 (2004).

[10] A. Błachowski, K. Ruebenbauer, J. Żukrowski, J. Alloys Comp. 464, 13 (2008).

[11] A. Błachowski, K. Ruebenbauer, J. Przewoźnik, J. Żukrowski, J. Alloys Comp. 458, 96 (2008).

[12] A. Błachowski, K. Ruebenbauer, J. Żukrowski, J. Przewoźnik, J. Alloys Comp. 455, 47 (2008).

[13] R. Górnicki, A. Błachowski, K. Ruebenbauer, Nukleonika 52, S7 (2007).

[14] S.M. Dubiel, W. Zinn, Phys. Rev. B 30, 3783 (1984).

[15] S.M. Dubiel, J. Magn. Magn. Mater. 69, 206 (1987).

[16] S.M. Dubiel, Phys. Rev. B 37, 1429 (1988).

[17] K. Królas, Phys. Lett. A 85, 107 (1981).

[18] A.Z. Hrynkiewicz, K. Królas, Phys. Rev. B 28, 1864 (1983).

[19] J. Chojcan, J. Alloys Comp. 264, 50 (1998).

[20] J. Chojcan, Hyperfine Interact. 156/157, 523 (2004). 